\title{
Linear tarification in multi-commodity telecommunications networks
}

Citation for published version (APA):

Bouhtou, M., van Hoesel, C. P. M., van der Kraaij, A. F., \& Lutton, J. L. (2002). Linear tarification in multicommodity telecommunications networks. METEOR, Maastricht University School of Business and Economics. METEOR Research Memorandum No. 012 https://doi.org/10.26481/umamet.2002012

Document status and date:

Published: 01/01/2002

DOI:

10.26481/umamet.2002012

Document Version:

Publisher's PDF, also known as Version of record

\section{Please check the document version of this publication:}

- A submitted manuscript is the version of the article upon submission and before peer-review. There can be important differences between the submitted version and the official published version of record.

People interested in the research are advised to contact the author for the final version of the publication, or visit the DOI to the publisher's website.

- The final author version and the galley proof are versions of the publication after peer review.

- The final published version features the final layout of the paper including the volume, issue and page numbers.

Link to publication

\footnotetext{
General rights rights.

- You may freely distribute the URL identifying the publication in the public portal. please follow below link for the End User Agreement:

www.umlib.nl/taverne-license

Take down policy

If you believe that this document breaches copyright please contact us at:

repository@maastrichtuniversity.nl

providing details and we will investigate your claim.
}

Copyright and moral rights for the publications made accessible in the public portal are retained by the authors and/or other copyright owners and it is a condition of accessing publications that users recognise and abide by the legal requirements associated with these

- Users may download and print one copy of any publication from the public portal for the purpose of private study or research.

- You may not further distribute the material or use it for any profit-making activity or commercial gain

If the publication is distributed under the terms of Article $25 \mathrm{fa}$ of the Dutch Copyright Act, indicated by the "Taverne" license above, 


\title{
Linear Tarification in Multi-Commodity Telecommunications Networks
}

\author{
Mustapha Bouhtou $^{*} \quad$ Stan van Hoesel ${ }^{\dagger} \quad$ Anton F. van der Kraaij ${ }^{\ddagger}$ \\ Jean-Luc Lutton $\S$
}

March 14, 2002

\begin{abstract}
We consider the problem of determining a set of optimal tariffs for a revenue maximizing operator, on a subset of all arcs of a telecommunications network. We suppose multiple rational clients are active on the network who route their demands on the cheapest paths from source to destination, where the cost of a path is determined by all costs and tariffs on the arcs of the path.

The complexity of the problem is studied first. Second, we propose a remodeling of the network, combined with model specific graph reduction methods. This new model is used in a branch and bound algorithm to solve the problem to optimality. Finally, we provide computational results which show the efficiency of our method for a number of real-life instances.

Keywords: Tarification, Telecommunications, Combinatorial Optimization, Branch \& Bound
\end{abstract}

\section{Introduction}

The market for telecommunications was until recently usually a monopolistic market, either a public monopoly as in the different European countries or a private monopoly as in the United States. In Europe, for several years deregularization of each national telecommunications market has taken place. As a consequence, multiple operators are active in the current telecommunications market. Each operator desires however to allow his clients to communicate with the clients of another network. Therefore, operators must decide among themselves what the price will be to exchange traffic on the connections between the operators, referred to as interconnections. The tariff-setting decision must be applied both with the operators at the national and international level. The determined price on the interconnections has an influence on the cost of routing the client's demand.

This tariff-setting problem involves two non-coöperative groups. On one side, are the operators who strive to set the tariffs on the interconnections of the network as high as possible in view of maximizing their revenue. On the other side, are the clients who wish to route their demands at minimum cost. As such, the route taken by each client in the network can involve interconnections belonging to different operators. The operator can only set the tariffs on the interconnections of the network which are owned by him and not by his competitors.

\footnotetext{
*France Télécom R\&D, 39-40 rue du Général Leclerc, F-92131 Issy-Les-Moulineaux, France. E-mail: mustapha.bouhtou@rd.francetelecom.fr

${ }^{\dagger}$ Dept of Quantitative Economics, Maastricht University, P.O.Box 616, 6200 MD Maastricht, The Netherlands. E-mail: s.vanhoesel@ke.unimaas.nl

$\ddagger$ Dept of Quantitative Economics, Maastricht University, P.O.Box 616, 6200 MD Maastricht, The Netherlands. E-mail: a.vanderkraaij@ke.unimaas.nl

$\S$ France Télécom R\&D, 39-40 rue du Général Leclerc, F-92131 Issy-Les-Moulineaux, France. E-mail: jeanluc.lutton@francetelecom.fr
} 
Game theory provides an adequate framework for this type of problem. For a reference, see Fisk [4] who proposes a model for the tariff-setting problem applied to the freight transportation problem. Through the selection of tariffs and levels of service, a Nash equilibrium is achieved by several competing carriers. In the case of road pricing in urban transportation networks, Ferrari [3] studied the use of tarification policies to influence network congestion and its negative externalities such as pollution.

In contrast with the game theoretical framework we consider the problem of determining the optimal revenue maximizing tariffs on a subset of all network arcs for a single operator. We suppose the tariffs set by all the other operators are known a priori. We assume furthermore that multiple rational clients route their demands on the network according to the shortest path from source to destination with respect to total cost.

For this type of problem, Labbé et al. [8] studied the tariffing of a multi-commodity transportation network. In the paper, they show that the problem is $\mathcal{N} \mathcal{P}$-hard and propose to model it as a bilevel model with bilinear objective functions. For this bilevel model, they furthermore prove that it can be reformulated as a mixed integer program. For the single-commodity case, primal-dual heuristics based on a penalization of the lower level objective function are proposed by Brotcorne et al. [1]. These heuristics are non-trivially extended to the multi-commodity case in Brotcorne et al. [2].

In the first part of the paper, we introduce the tarification problem and state some models previously defined in the literature. Subsequently, we address the complexity of the tarification problem studied. In the second part we propose a remodeling of the network, combined with model specific graph reduction methods. This remodeling is used in a branch and bound algorithm to solve the tarification problem occurring when dealing with multiple operators and multiple clients in the network.

Finally, we present some numerical results which show the efficiency of our method and conclude the paper.

\section{Tarification Problem}

In this section we define the tarification problem. We consider a telecommunications network represented by a directed graph $G=(N, A)$ with nodes $N$ and $\operatorname{arcs} A$, where the set $A$ is composed of the two disjoint sets $T$ and $F$. On this network, multiple operators and multiple clients are active. We restrict our attention to one operator who maximizes his revenue from the tariffs on the $\operatorname{arcs}$ in $T$, referred to as tariff arcs. For each tariff arc, the tariff corresponds to a revenue generating toll for routing one unit of a client's demand. The other arcs in the network correspond to the arcs of the other operators in the network, whose tariffs are known a priori and hence can be viewed as fixed costs on the arcs. We refer to these arcs by the set of fixed $\operatorname{arcs} F$.

The cost of routing a unit demand on an arc, both for the tariff arcs and for the fixed arcs is assumed to be nonnegative. In urban transportation literature, negative tariffs are sometimes allowed and represent, for example, subsidies given by the government owning the toll roads. However, in telecommunications, such practice is not established among operators of a network and is therefore not accounted for in our model. The clients on the network route their demands from source to destination according to the shortest path with respect to total cost, where the total cost of a path is defined as the sum of all the tariffs and fixed costs on the arcs of the path. Whenever the client can choose among multiple shortest paths with the same total cost but with different revenues for the operator, we suppose the client takes the shortest path that is most profitable to the operator.

We denote by $c_{a}$ the cost of routing a unit demand on a fixed arc $a \in F$ and by $t_{a}$, to be determined by the operator, the cost of routing a unit demand on a tariff $\operatorname{arc} a \in T$. The set of all paths that connect the source and destination of all clients $k \in K$ are given by $P_{k}$. Furthermore, let the cost of a path $p \in P_{k}$, as a function of all tariffs, be given by the function $c_{p}(t)$. Similarly, refer to the revenue for the operator associated with a path $p \in P_{k}$ by $\pi_{p}(t)$.

The reader may note that our model implicitly incorporates arcs with both fixed and tariff 
costs since we can split a tariff $\operatorname{arc} a$ with cost $c_{a}$ and an additional tariff $t_{a}$ into two arcs: a fixed arc with cost $c_{a}$ and a tariff arc with tariff $t_{a}$. We therefore assume that the tariff arcs only have tariffs assigned to them.

To ensure that the problem is bounded, we assume that there always exists a path for each client from source to target which uses only fixed arcs. Otherwise, the operator can set the tariff on at least one tariff arc arbitrarily high.

We can formulate the tarification problem as follows.

$$
\begin{array}{ll}
\max & \sum_{k \in K} \pi_{p_{k}^{*}}(t) \\
\text { s.t. } & p_{k}^{*} \in \arg \min _{\forall p \in P_{k}} c_{p}(t) \quad \forall k \in K
\end{array}
$$

The formulation given by equation (1) is a bilevel problem where at the upper level the operator strives to maximize his revenue, while at the lower level the clients seek to minimize the cost of routing their demands. Both objective functions are linear and hence we are dealing with a linearlinear bilevel program. The linear-linear bilevel program has been shown to be $\mathcal{N} \mathcal{P}$-Complete by Jeroslow [6]. For a reference on bilevel programming, we refer the reader to Vicente and Calamai [9] who have compiled an annotated bibliography on this subject containing more than one hundred references. Notice furthermore that the bilevel program given by equation (1) is not polynomial in its input data, since the set of all possible paths for each client $k \in K$ is exponential.

Let the vector $b^{k}$ be the demand/supply vector for each commodity where each element of the vector represents the demand/supply for a commodity at each node in the graph. As shown by Labbé et al. [8], we can rewrite equation (1) to the following bilevel model.

$$
\begin{array}{lll}
\max _{t} & t^{T} \sum_{k \in K} y_{1}^{k} & \\
\min _{y_{1}^{k}, y_{2}^{k}} & t^{T} \sum_{k \in K} y_{1}^{k}+c_{2}^{T} \sum_{k \in K} y_{2}^{k} & \\
\text { s.t. } & A_{1} y_{1}^{k}+A_{2} y_{2}^{k}=b^{k} & \forall k \in K \\
& y_{1}^{k}, y_{2}^{k} \geq 0 & \forall k \in K \\
& t \geq 0 &
\end{array}
$$

In this bilevel model, $y_{1}^{k} \in \mathbb{R}^{|T|}$, resp. $y_{2}^{k} \in \mathbb{R}^{|F|}$ represents the flow on the tariff, resp. fixed arcs, in vector notation. The costs on the arcs in the set $F$ are denoted by the vector $c_{2} \in \mathbb{R}^{|F|}$, while the vector of tariffs for the $\operatorname{arcs}$ in the set $T$ is given by $t \in \mathbb{R}^{|T|}$. The matrix $A_{1}$, resp. $A_{2}$, represents the node-arc incidence matrix for the tariff, resp. fixed arcs.

For the bilevel model described in equation (2), Brotcorne et al. [1] have developed primal-dual heuristics for the single-commodity case. These heuristics are extended to the multi-commodity case by Brotcorne et al. [2].

Labbé et al. [8] have shown that it is possible to formulate the bilevel model described in equation (2) as a mixed integer program. To arrive at this formulation, subject each commodity to a tax level $t^{k}$ and force equality of all tax levels for all commodities. Furthermore, for a given commodity flow, perform the change of variables $y_{i}^{k}=d^{k} \Phi_{i}^{k}$ for $i=\{1,2\}$, where $d^{k}$ indicates the demand of client $k \in K$ and $\Phi_{i}^{k} \mid i=\{1,2\}$ is a vector whose binary elements indicate whether or not a flow passes on a certain arc. As shown by Labbé et al. [8], we can replace the lower level program by its optimality conditions (duality-gap is zero). This yields the following mixed integer program, referred to in the remainder of this paper as MIP. 


$$
\begin{array}{clr}
\max _{t, t^{k}, \Phi_{1}^{k}, \Phi_{2}^{k}, \lambda^{k}} & \sum_{k \in K}\left(e^{T} t^{k}\right) d^{k} & \\
\text { s.t. } & A_{1} \Phi_{1}^{k}+A_{2} \Phi_{2}^{k}=b^{k} / d^{k} & \forall k \in K \\
& \Phi_{1}^{k}, \Phi_{2}^{k} \geq 0 & \forall k \in K \\
& A_{1}^{T} \lambda^{k} \leq t & \forall k \in K \\
& A_{2}^{T} \lambda^{k} \leq c_{2} & \forall k \in K \\
& e^{T} t^{k}+\epsilon^{\Phi_{2}^{k}}=\left(b^{k} / d^{k}\right)^{T} \lambda^{k} & \forall k \in K \\
& -M \Phi_{1}^{k} \leq t^{k} \leq M \Phi_{1}^{k} & \forall k \in K \\
& -M\left(1-\Phi_{1}^{k}\right) \leq t^{k}-t \leq M\left(1-\Phi_{1}^{k}\right) & \forall k \in K \\
& \Phi_{1}^{k}, \Phi_{2}^{k} \in\{0,1\} & \forall k \in K \\
& t \geq 0 &
\end{array}
$$

For this mixed integer program formulation, the vector $e$ denotes the unit vector, whose coefficients are all equal to one. We will use an industry standard ILP solver (CPLEX 7.5) to solve the mixed integer program and use its results as a benchmark for the branch-and-bound algorithm proposed in this paper.

\section{Complexity}

Labbé et al. [8] studied the tarification problem on a transportation network and have shown that the related decision problem is $\mathcal{N} \mathcal{P}$-complete for a single commodity when a lower bound on the tariffs is given. In this paper we propose an $\mathcal{N} \mathcal{P}$-completeness proof for the single commodity tarification problem without lower bounds on the tariffs, which is an adaptation of the proof given by Labbé et al. [8]. To this end, we first consider the following lemma.

Lemma 1. If for an assignment of tariffs the revenue of an operator is nonnegative, then the path taken by the client in the network contains no cycles.

Proof. Suppose the path taken by the client contains cycles. Positive cycles cannot occur, since this is not in the client's interest. Hence, we can assume that there is an assignment of tariffs such that negative cycles on the path occur. Since the cost of an arc is assumed to be nonnegative, the sum of all tariffs on the cycle is negative. Hence, the client can go through the cycle an infinite amount of times, making his path cheaper while decreasing the profit of the operator infinitely.

Denote the decision problem of the tarification problem (TP) as follows:

\section{TP}

INSTANCE: A directed graph $G=(N, A)$, a set of tariff $\operatorname{arcs} T \subseteq A$, a cost $c_{a} \geq 0$ for each arc $a \in A$, a variable $t_{a}$ for each tariff arc $a \in T$, and a bound $B \in \mathbb{Z}^{+}$.

QUESTION: Is there an assignment of tariffs $t_{a}$ for the tariff $\operatorname{arcs} a \in T$ such that when the client takes the shortest path $p$ from $s$ to $t$ :

$$
\sum_{a \in\{T \cap p\}} t_{a} \geq B \quad ?
$$

Theorem 1. TP is NP-Complete.

Proof. It is easy to see that $T P \in N P$, because a non-deterministic algorithm need only guess a tarification scheme and can check in polynomial time if the shortest path taken by the client yields cumulative tariffs of at least $B$.

We transform Hamiltonian Path on a directed graph (see Garey and Johnson [5]) to TP. Let an arbitrary instance of Hamiltonian Path be given by the directed graph $G=(N, A)$. We must 
construct a graph $G^{*}=\left(N^{*}, A^{*}\right)$ such that $T P$ on $G^{*}$ has a solution if and only if $G$ has a Hamiltonian Path. Let $G^{*}$ be a copy of the graph $G$ with $T=A$, cost equal to -1 for all arcs and an additional non-tariff arc $s-t$ with cost $|N|-1$. We set the bound $B$ to $2(|N|-1)$. If $G$ has a Hamiltonian Path, then a solution to $T P$ is constructed by setting the tariff on the arcs of the Hamiltonian path from $s$ to $t$ to 2 and to $|N|$ on all other arcs of the graph $G^{*}$. The revenue from tariffs is clearly $2(|N|-1)$.

We show the reverse by proving that if $G$ does not have a Hamiltonian Path then a solution to $T P$ with $B=2(|N|-1)$ does not exist. Denote by $R$ the revenue from tariffs on the shortest path from $s$ to $t$. Let $k$ be the number of arcs on the path $p$. Any tarification scheme must satisfy $R-k \leq|N|-1$. Thus $R \leq|N|-1+k$. For $R$ to be at least $2(|N|-1)$, we must have that $k \geq|N|-1$. By lemma 1, we know that negative cycles cannot occur. Hence, $k \geq|N|-1$ is impossible, since we would then have a Hamiltonian Path in $G$.

Consider another variant of the tarification problem, and refer to it as the set tarification problem. For this problem, where we are dealing with one client and multiple tariff arcs, the set of tariff arcs with positive flow in the solution is known a priori and we can show that it is $\mathcal{N} \mathcal{P}$-hard. We define its related decision problem (STP) as follows:

\section{STP}

INSTANCE: A directed graph $G=(N, A)$, a set of tariff $\operatorname{arcs} T \subseteq A$, a subset of the tariff $\operatorname{arcs}$ $T^{*} \subseteq T$, a cost $c_{a}$ for each arc $a \in A$, a variable $t_{a}$ for each tariff arc $a \in T$, and a bound $B \in \mathbb{Z}^{+}$. QUESTION: Is there an assignment of tariffs $t_{a}$ for the tariff $\operatorname{arcs} a \in T$ such that when the client takes the shortest path $p$ from $s$ to $t$ going through the tariff $\operatorname{arcs}$ in the set $T^{*}$ :

$$
\sum_{a \in\{T \cap p\}} t_{a}=\sum_{a \in\left\{T^{*} \cap p\right\}} t_{a} \geq B \quad ?
$$

Theorem 2. STP is $\mathcal{N} \mathcal{P}$-complete.

Proof. Analogous to the proof of theorem 1 we can easily see that $S T P \in \mathcal{N} \mathcal{P}$.

We transform Hamiltonian Path on a directed graph (DHP, see Garey and Johnson [5]) to the Set Tarification Problem (STP).

Since all arcs in the network have costs assigned to them, the cost of routing a unit demand on an arc is equal to the sum of the cost and the possible tariff on the arc. The reader may note that for all possible paths going through the tariff arcs in $T^{*}$, the sum of all tariffs is the same. Hence, it is the cost of the fixed arcs on the path that determines which path the client takes.

Let an arbitrary instance of Hamiltonian Path be given by the directed graph $G=(N, A)$. We construct a graph $G^{*}=\left(N^{*}, A^{*}\right)$ such that $S T P$ on $G^{*}$ has a solution if and only if $G$ contains a Hamiltonian Path.

Let $G^{*}$ be the polynomial transformation of the graph $G$ where each node $i \in N$ has been replace by the two nodes $i^{-}$and $i^{+}$. The node $i^{-}$in $G^{*}$ contains all the in-arcs of the original graph $G$ for $i \in N$ and $i^{+}$all the out-arcs of the original graph $G$ for $i \in N$. Moreover, for each node $i \in N$ we create a tariff arc $\left(i^{-}, i^{+}\right)$in $G^{*}$ with cost zero. All arcs in $G$ correspond to tariff arcs in $G^{*}$ with cost equal to 1 . The set $T$ corresponds to all the defined tariff arcs in $G^{*}$, while the set $T^{*}$ consists of those tariff arcs created from the nodes in $N$. A hamiltonian path in the graph $G$ from $s$ to $t$ corresponds to a unit demand from $s^{-}$to $t^{+}$in the graph $G^{*}$. Finally, we create an additional non-tariff arc $\left(s^{-}, t^{+}\right)$with cost $(|N|-1)$ in $G^{*}$ and set the bound $B$ to zero. For a graphical representation of the transformation, see also figure 1 .

Given a hamiltonian path in $G$, a solution to $S T P$ is constructed by setting the tariff on the arcs corresponding to the nodes of the hamiltonian path from $s$ to $t$ and the tariff on arcs corresponding to the arcs of the hamiltonian path to zero. For all other tariff arcs we set the tariff to $|N|$. The shortest path from $s^{-}$to $t^{+}$is the path going through all tariff arcs with cost 1 and all tariff arcs with cost of zero, yielding a total cost of $|N|-1$ and a revenue of zero.

Given is an optimal tarification scheme for which the client takes the path $p$ yielding revenue of zero and going through all tariff arcs in $T^{*}$. Total revenue is zero, the maximal cost of the 

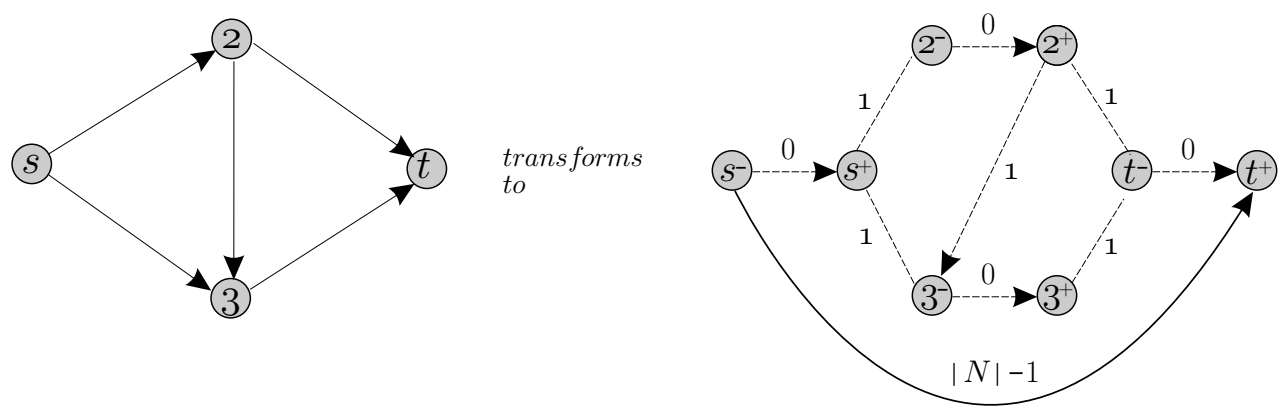

Figure 1: DHP to STP transformation.

path $p$ is $(|N|-1)$ and $\left|T^{*}\right|=|N|$, hence the cost of the path $p$ is equal to $|N|-1$. By lemma 1 , cycles on the path $p$ due to a particular tarification scheme cannot occur. We can thus retrieve a hamiltonian path in $G$ by going through the nodes in $G$ in the same sequence as the corresponding tariff arcs on the path $p$ in $G^{*}$.

Some variants of the tarification problem have been shown to be solvable by polynomial-time algorithms. Among these is the class of problems with only a single tariff arc and multiple clients and the class of problems where the operator is dealing with multiple tariff arcs and a single client for which the path taken at optimality is known a priori. The reader is referred to Labbé et al. [8] for a description of these algorithms.

To these polynomially solvable variants of the tarification problem we can add the problem referred to as the equal tariffs problem (ETP). For this problem we are dealing with multiple clients and multiple tariff arcs for which the operator must determine the tariffs such as to maximize his revenue. All tariffs as determined by the operator must however be the same on all tariff arcs.

Proposition 1. ETP is polynomially solvable.

When all tariffs to be determined must be equal, we can calculate for each client all the tariff values for which the shortest path tree changes by using a polynomial time parametric shortest path algorithm as described by Young and Faster [10] and Karp and Orlin [7]. Denote all these tariff values by $t_{1}, \ldots, t_{n}$. Furthermore, define the revenue generated by client $k \in K$ as a function of the tariff $t$ on the $\operatorname{arcs}$ by $\pi_{k}(t)$ and let

$$
i^{*} \in \arg \max _{i}\left\{\sum_{k \in K} \pi_{k}\left(t_{i}\right): i=1, \ldots, n\right\}
$$

The optimal tarification scheme is given by setting the tariffs on all tariff $\operatorname{arcs}$ to $t_{i^{*}}$.

\section{Shortest Paths Graph Model}

In this section, we describe for each commodity a graph remodeling of the network referred to as the shortest paths graph model (SPGM).

In a solution to the tarification problem a client will always take the shortest path between two nodes. Hence, if he does not take any tariff arc between two nodes he takes the shortest path using only fixed cost arcs between two nodes. Therefore, when a client takes a path containing two tariff arcs, two situations can occur: either there is a shortest path consisting only of fixed cost arcs between the two tariff arcs or the head node of the first tariff arc is the tail node of the second tariff arc on the path. In the latter case, we can suppose without loss of generality that there exists a shortest path using only fixed arcs with cost zero between the two tariff arcs.

The shortest paths graph model is as follows. Given is the original graph $G=(N, A)$ with the tariff $\operatorname{arcs}$ in $T \subseteq A$. For each client with a demand from $s$ to $t$, define the graph $G^{*}=\left(N^{*}, A^{*}\right)$ 


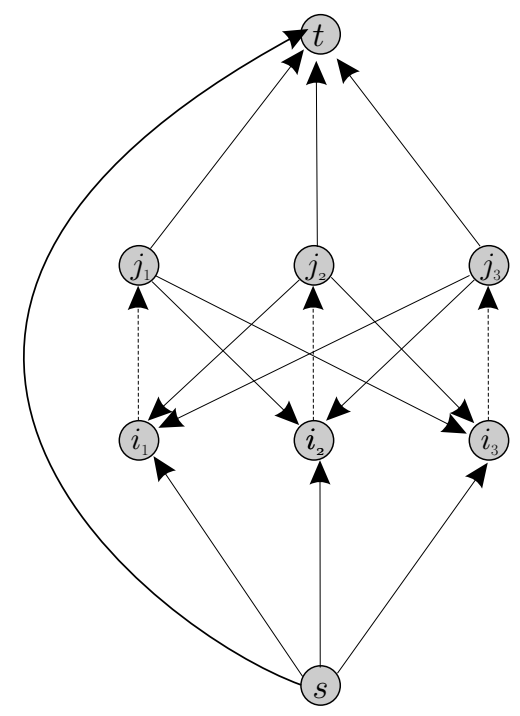

Figure 2: Shortest Paths Graph Model for $|T|=3$.

and tariff $\operatorname{arcs} T^{*} \subseteq A^{*}$. In this graph $N^{*}=\{s\} \cup\{t\} \cup\{i \in N \mid(i, j) \in T\}$ and $T^{*}=T$. The fixed $\operatorname{arcs} F^{*} \subseteq A^{*}$ are representations of the cost of the shortest paths using only fixed arcs in the original network from the source node to the tails of the tariff arcs, the head nodes of the tariff arcs to the target node, the head node of each tariff arc to the tail nodes of the other tariff arcs and finally from the source node to the target node. By this transformation we obtain a graph $G^{*}$ containing all possible paths in $G$ from $s$ to $t$ using all possible alternating sequences of tariff and fixed arcs.

Example 1. Figure 2 shows the shortest paths graph model of any network containing three tariff arcs for a commodity from $s$ to $t$. The tariff arcs are given by the $\operatorname{arcs}\left(i_{1}, j_{1}\right),\left(i_{2}, j_{2}\right)$ and $\left(i_{3}, j_{3}\right)$. All other arcs are representations of the shortest path using no tariff arcs between each node. The cost of the arc is the cost of the corresponding shortest path in the original network between the two nodes. If no path exists between two nodes in the original network, the corresponding arc in the shortest paths graph model is not present or has infinite cost.

For the shortest paths graph model we can define the inner graph, consisting of the nodes of the tariff arcs, the tariff arcs and the fixed arcs between each head and tail node of the tariff arcs. All the paths (represented by arcs) in the inner graph need to be calculated only once for all commodities, since they are the same for each commodity. The other paths, from the source node to the tail nodes of the tariff arcs, from the head nodes of the tariff arcs to the target node and from the source node to the target node, may differ for each commodity.

\section{Reduction Methods}

The purpose of the new model for the network proposed in section 4 is to obtain a graph which contains few relevant paths for each commodity. We can reduce the size of the shortest paths graph model considerably by using arc reduction methods. Path reduction methods allow us to reduce the set of relevant paths even more. In the next subsections we discuss these reduction methods.

\subsection{Arc Reductions}

We will describe the arc reduction methods by the following propositions. Denote by $d_{i j}$, the cost of the shortest path using only fixed arcs from node $i$ to node $j$ and let $l_{i j}$ denote the cost of the 


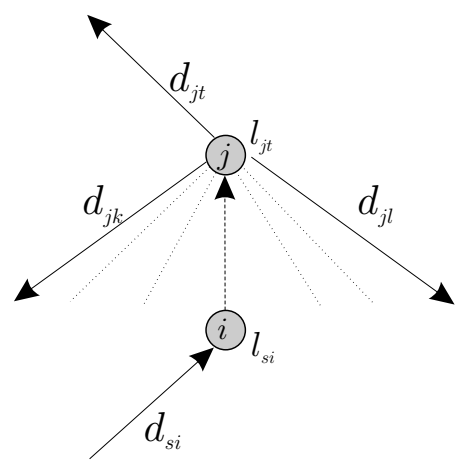

Figure 3: Detailed view of tariff arc

shortest path, possibly using tariff arcs, when the tariffs are set to zero from node $i$ to node $j$. For each commodity, node $s$ represents the source node and node $t$ the target node. See also figure 3 for a detailed view of a tariff arc.

Since $l_{i j}$, compared to $d_{i j}$, is the cost of a shortest path in a graph with extra arcs of zero cost we can state that $l_{i j}$ is a lower bound for the cost of a path from $i$ to $j$, whereas $d_{i j}$ is an upper bound for the cost of a path from $i$ to $j$ taken by the client.

Proposition 2. If $l_{j t}=d_{j t}$, then node $j$ of the tariff arc $(i, j)$ has no outgoing arcs $(j, k)$, where $k$ is the tail of another tariff arc.

Proof. A lower bound on the cost of any path from $j$ to $t$ going through the other tariffs arcs is $l_{j t}$. Hence there is room for taxation on paths from $j$ to $t$ using other tariff arcs only if $l_{j t}<d_{j t}$.

Proposition 2 is especially relevant when the shortest path with all tariffs set to zero from node $j$ of a tariff arc $(i, j)$ to node $t$ uses only fixed arcs. By symmetry, we also have the following proposition.

Proposition 3. If $l_{s i}=d_{s i}$, then node $i$ of the tariff arc $(i, j)$ has no incoming arcs $(n, i)$, where $n$ is the head of another tariff arc.

Proof. Analogous to proposition 2.

Proposition 4. If $d_{j t} \leq d_{j k}+l_{n t}$, we can delete for a tariff arc $(i, j)$ the outgoing arc $(j, k)$, where $k$ is the tail of another tariff arc $(k, n)$.

Proof. The lower bound on the cost of a path from $j$ to $t$ taking the $\operatorname{arc}(j, k)$ is equal to $d_{j k}+l_{n t}$. An upper bound on the cost of a shortest path is given by $d_{j t}$. Hence, there is room for taxation on a path from $j$ to $t$ using the $\operatorname{arc}(j, k)$ only if $d_{j t}>d_{j k}+l_{n t}$.

Proposition 5. If $d_{s i} \leq d_{n i}+l_{s k}$, we can delete for the tariff arc $(i, j)$ the incoming arc $(n, i)$, where $n$ is the head of another tariff arc $(k, n)$.

Proof. Analogous to proposition 4.

Proposition 6. If $d_{s t} \leq d_{j k}+l_{n t}+l_{s i}$, we can delete for the tariff arc $(i, j)$ the outgoing arc $(j, k)$, where $k$ is the tail of another tariff arc $(k, n)$.

Proof. The upper bound on the cost of a shortest path from $s$ to $t$ is $d_{s t}$. A lower bound on the cost of a path using the tariff arc $(i, j)$ and going to the tariff $\operatorname{arc}(k, n)$ is at $l_{s i}+d_{j k}+l_{n t}$. Hence, there is room for taxation on a path from $s$ to $t$ using the $\operatorname{arc}(j, k)$ only if $d_{s t}>d_{j k}+l_{n t}+l_{s i}$.

Proposition 7. If $d_{s t} \leq l_{s i}+l_{j t}$, we can delete the tariff arc $(i, j)$. 

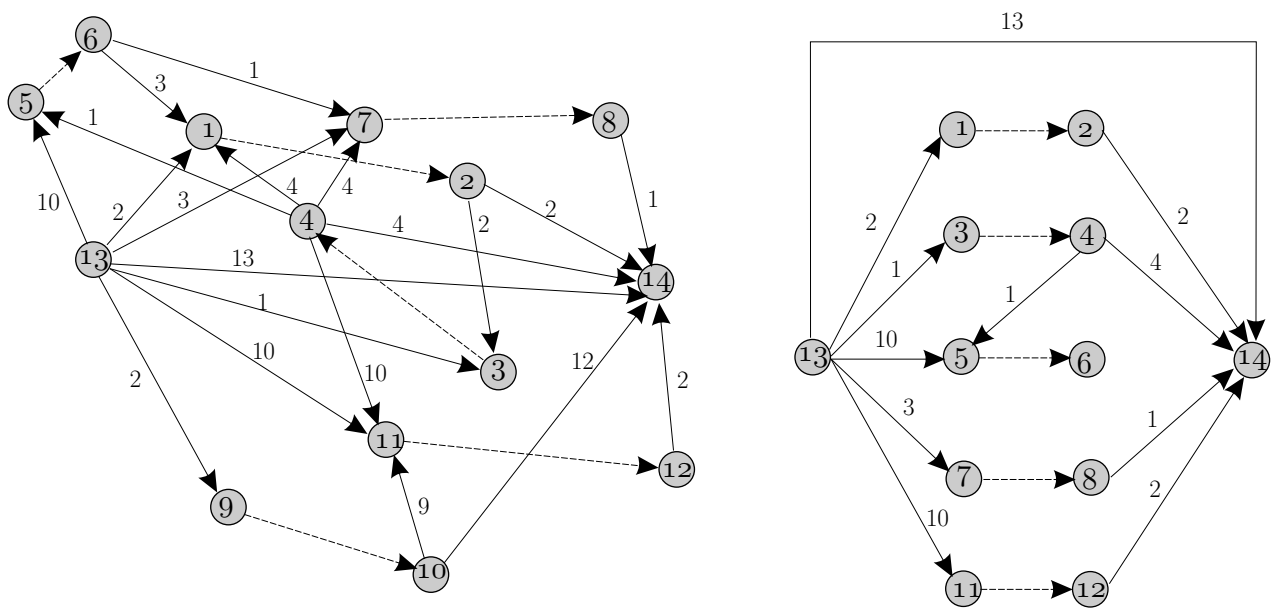

Figure 4: Original graph (left) and final SPGM graph (right).

Proof. The lower bound on the cost of a path going through the tariff arc $(i, j)$ is $l_{s i}+l_{j t}$. Hence, there is room for taxation on the tariff arc $(i, j)$ only if $d_{s t}>l_{s i}+l_{j t}$.

Example 2. We can illustrate each proposed reduction method on the graph shown in figure 4 on the left. For this instance, we are dealing with one client who has a unit demand from node 13 to node 14. The tariff arcs in the network are indicated by the dotted arcs. Propositions 2 and 3 allow us to delete for the tariff arc $(1,2)$ all the incoming arcs of node 1 and all the outgoing arcs of node 2 . We can delete the arcs $(4,7)$ and $(4,11)$ by either applying proposition 4 or proposition 5 . By applying proposition 5 or proposition 6 we can delete the outgoing arc $(10,11)$ of the tariff arc $(9,10)$. Proposition 7 allows us to delete the tariff arc $(9,10)$ from the graph.

The final graph is shown on the right in figure 4. For this graph it is easy to see that the optimal solution is to set the tariff on the arc $(1,2)$ to 9 , while setting all the other tariffs to a suitable large value, i.e. 13, yielding a revenue of 9 for the operator.

\subsection{Path Reductions}

By applying the propositions described in section 5.1 and finding all paths in the graph, we can already obtain a set of paths which are relevant for the commodity. Hopefully, this set of paths is small. It is however possible to reduce the size of this set in some instances even more by eliminating paths which are always dominated by others. The notion of path dominance is given by the following definition.

Definition 1. If we can replace in all feasible solutions the path $p_{2}$ by the path $p_{1}$ without violating the feasibility constraints or changing the value of the objective function, then path $p_{1}$ dominates path $p_{2}$.

The following proposition allows us to eliminate dominated paths.

Proposition 8. Consider a path $p_{1}$, resp. $p_{2}$, with tariff arcs in $T_{1} \subseteq T$, resp. $T_{2} \subseteq T$, and with total cost $c_{1}$, resp. $c_{2}$, when all tariffs are zero. If $T_{1} \subseteq T_{2}$ and $c_{2} \geq c_{1}$, then path $p_{1}$ dominates path $p_{2}$ for all tariff values.

Proof. Denote by $t_{a}$ the tariff on $\operatorname{arc} a$ and suppose that path $p_{2}$ is the shortest path taken by the client. Then it must hold for all feasible solutions:

$$
c_{2}+\sum_{a \in T_{2}} t_{a} \leq c_{1}+\sum_{a \in T_{1}} t_{a} \Leftrightarrow c_{2}+\sum_{a \in\left\{T_{2} \backslash T_{1}\right\}} t_{a} \leq c_{1}
$$




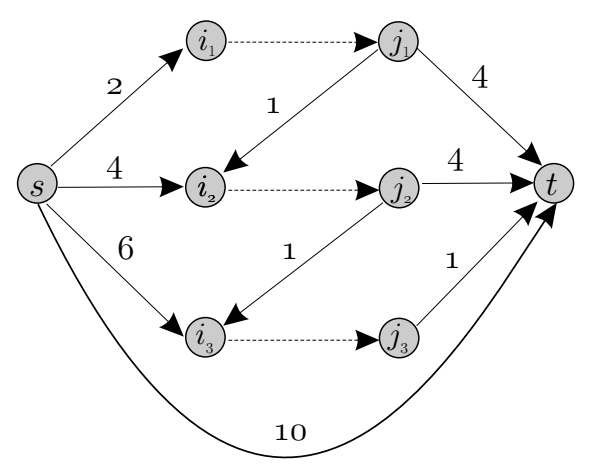

Figure 5: Dominated paths example.

Since $c_{2} \geq c_{1}$ and $t_{a} \geq 0$ for all tariff $\operatorname{arcs} a \in A$, equation 5 only holds when $c_{2}=c_{1}$ and $t_{a}=0$ for all $a \in\left\{T_{2} \backslash T_{1}\right\}$. Hence, we can replace path $p_{2}$ by path $p_{1}$ without violating the feasibility constraints or changing the value of the objective function since path $p_{1}$ has the same revenue for the operator and the same cost for the client as path $p_{2}$. Equation 5 shows furthermore that whenever $c_{2}>c_{1}$, path $p_{2}$ can never be the path taken by a client in a feasible solution, since path $p_{1}$ will always be cheaper.

Example 3. An instance where this dominance of paths occurs is given in figure 5 . The tariff arcs are the $\operatorname{arcs}\left(i_{1}, j_{1}\right),\left(i_{2}, j_{2}\right)$ and $\left(i_{3}, j_{3}\right)$. The operator is dealing with one client who wants to route his demand from node $s$ to node $t$. For this graph, the path $\left\{s, i_{1}, j_{1}, i_{2}, j_{2}, t\right\}$ is dominated by the path $\left\{s, i_{1}, j_{1}, t\right\}$.

The shortest paths graph model combined with the arc and path reduction mentioned in this section allow us to create a sparse graph for each commodity, which in turn yields a small set of relevant paths for each commodity, as will be shown by the numerical results in section 7 .

\section{Branch and Bound Algorithm}

In this section we will derive a branch and bound algorithm for our tariff-setting problem which uses the previously mentioned shortest paths graph model and reduction methods. Although the lower and upper bounding methods used are relatively simple, our numerical results (see section 7) will show that the algorithm is very effective.

The branch and bound algorithm consists of two steps. The first step is a preprocessing step, where we create for each client a shortest paths graph model and apply to it the reduction methods of section 5. For each client we can subsequently find all the relevant shortest paths in the graph. Of course, having all the relevant shortest paths for each client is not enough. We still need to know which path each client takes in the optimal solution. It is this optimization problem we solve in step two of the algorithm by a classical branch and bound method.

As stated in section 2, denote the clients by the set $K$ and the set of all paths a client $k \in K$ can take by $P_{k}$. The reduction methods applied to the shortest paths graph model allow us to determine the set of relevant paths for each commodity. Without loss of generality we suppose that the set $P_{k}$ consists only of these relevant paths. Denote furthermore by the linear function $c_{p}(t)$ the cost of a path $p$ as a function of all tariff values and by the linear function $\pi_{p}(t)$ the associated revenue for the operator on the path $p$.

When we fix the path taken by a client $k$ a priori, and denote it by $p_{k}^{*}$, we can find the optimal, revenue maximizing tariffs such that the client indeed takes the path $p_{k}^{*}$ by solving a linear problem of the following type: 


$$
\begin{array}{ll}
\max & \sum_{k \in K} \pi_{p_{k}^{*}}(t) \\
\text { s.t. } & c_{p}(t) \geq c_{p_{k}^{*}}(t) \quad \forall k \in K, \forall p \in P_{k}
\end{array}
$$

The linear program described in equation (6) ensures that the path $p_{k}^{*}$ is indeed the shortest path in $P_{k}$, while maximizing the operator's revenue. As will be shown by the numerical results, when we use the shortest paths graph model and apply to it the reduction methods previously mentioned, we can obtain small-sized sets $P_{k}$ which makes the number of constraints for the previous linear program small.

\subsection{Node Processing}

Due to our branching rules, which we will describe in section 6.2 , we have that in each node of the branch and bound tree for some clients the path taken in the solution is fixed, whereas for other clients this is not the case. Therefore, in each node, we denote by the set $K_{f} \subseteq K$ the set of clients for which we have fixed the path taken in the solution. With this information we can generate lower and upper bounds on the total revenue generated by the clients for the operator by solving linear programs as described in equation (6) in each node of the tree.

We generate lower bounds in each node of the branch and bound tree by generating a feasible solution. Hence, we have to fix for all clients the path taken in the solution. For each client $k \in K_{f}$ we already know which path they take in the solution and we denote it by $p_{k}^{*}$. For the other clients we have not (yet) fixed their paths. Therefore, in each node of the tree, for each client for which a path is not fixed, i.e. all clients in $\left\{K \backslash K_{f}\right\}$, we make an assumption on the path taken: we set the path taken to the path with smallest fixed cost and denote this path by $l_{k}$.

$$
\begin{array}{lll}
\max & \sum_{k \in K_{f}} \pi_{p_{k}^{*}}(t)+\sum_{k \in\left\{K \backslash K_{f}\right\}} \pi_{l_{k}}(t) & \\
\text { s.t. } & c_{p}(t) \geq c_{p_{k}^{*}}(t) & \forall k \in K_{f}, \forall p \in P_{k} \\
& c_{p}(t) \geq c_{l_{k}}(t) & \forall k \in\left\{K \backslash K_{f}\right\}, \forall p \in P_{k} \\
& t_{a} \geq 0 & \forall a \in T
\end{array}
$$

A lower bound in a node can subsequently be obtained by solving a linear program as described in equation (7). If the linear program is infeasible, we set the lower bound of the node to minus infinity.

Before we describe how to calculate an upper bound on the total revenue for the operator, we show how to calculate an upper bound on the revenue generated by a single client.

A client will not accept a cost that is higher than the cost of a shortest path when the tariffs are set to infinity. Denote this path for each client $k \in K$ by the path $u_{k}$. Furthermore, since the tariffs and costs are restricted to be nonnegative, the minimal cost of a shortest path for a client is the cost of a shortest path when the tariffs are set to zero. This corresponds to the path with minimal fixed cost for each client, which we have previously denoted by $l_{k}$ for each client $k \in K$. Therefore, for a client $k \in K$ an upper bound for the revenue generated by that client is given by $\left[c_{u_{k}}(t)-c_{l_{k}}(t)\right]$, where $c_{u_{k}}(t)$, resp. $c_{l_{k}}(t)$, indicates the cost of the shortest path of client $k$ from source to destination with tariffs at infinity, resp. zero. As is shown by Labbé et al. [8], this upper bound is not necessarily reached. This can easily be verified by the example given in figure 6. For a single client with a unit demand from node 1 to node 4 , the optimal tarification scheme is to set the tariffs on the tariff arcs to $t_{1}=t_{2}=2$, yielding a revenue of 4 . The upper bound on the revenue is however given by $7-2=5$.

In each node of the branch and bound tree, let $\Pi^{*}$ be the revenue generated by the demands which are fixed, i.e. the demands $k \in K_{f}$. We can calculate $\Pi^{*}$ by solving the following linear program. 


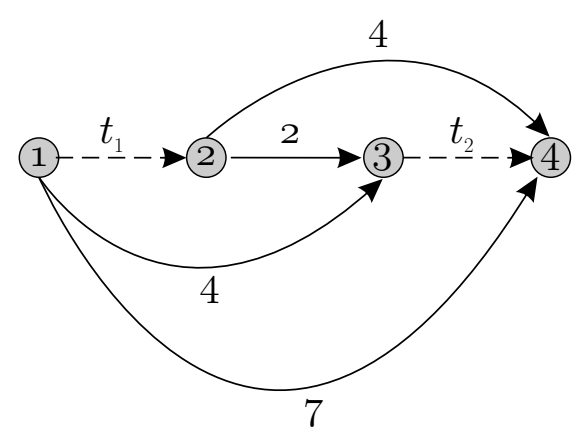

Figure 6: Upper bound on revenue is not reached.

$$
\begin{array}{lll}
\max & \sum_{k \in K_{f}} \pi_{p_{k}^{*}}(t) & \\
\text { s.t. } & c_{p}(t) \geq c_{p_{k}^{*}}(t) & \forall k \in K_{f}, \forall p \in P_{k} \\
& t_{a} \geq 0 & \forall a \in T
\end{array}
$$

Clearly, the clients in the set $K_{f}$ generate a revenue of $\Pi^{*}$. Given the upper bound on the revenue for each single client, an upper bound on the revenue generated by all the clients for which the paths are not fixed is given by:

$$
\sum_{k \in K \backslash K_{f}}\left\{c_{u_{k}}(t)-c_{l_{k}}(t)\right\}
$$

An upper bound on the total revenue for the operator in a node is thus given by:

$$
\Pi^{*}+\sum_{k \in K \backslash K_{f}}\left\{c_{u_{k}}(t)-c_{l_{k}}(t)\right\}
$$

This upper bound omits however all the information we can retrieve from the constraints of the linear program given by equation (8). Consider the constraints of the linear program whose variable coefficients are either 0 or 1 . These constraints are of the following form:

$$
\sum_{u \in U_{i}^{k}} t_{u} \leq c_{i}
$$

where for a client $k \in K$ and a row $i$ of the constraint matrix of the linear program given by equation (8), the set $U_{i}^{k}$ denotes those tariff arcs whose coefficients in the constraint are all equal to 1 . The right hand side of the constraint $i$ is given by the constant $c_{i}$, whereas the variables associated with each tariff arc are denoted by $t_{u}$. If a client takes the path $u_{k}$, the upper bound on the revenue is nil. Hence, consider each client that does not take the path $u_{k}$. For these clients, we know there is at least one constraint as given by equation (11) since we must have that the cost of the selected path is less or equal to the cost of the path $u_{k}$, which is a constant. Depending on the instance we are dealing with, it is of course possible to have more than one such constraint for a client.

Without loss of generality, denote by $\Phi=\{1 \ldots m\}$ the set of constraints which are of the form given by equation (11). We can retrieve these constraints from the linear program in equation (8), which corresponds to the constraints of the clients $k \in K_{f}$ whose paths are already fixed in a certain node $n$ of the branch and bound tree. For all the child nodes of the node $n$, these constraints provide upper bounds on the tariffs for the paths taken by the clients $k \in\left\{K \backslash K_{f}\right\}$. Let $\tau_{p}$ be the set of tariff $\operatorname{arcs}$ on the path $p \in P_{k}$ for a client $k \in K$. We can now improve the previous upper bound on the cost of a path $p$, which we denoted by $c_{u_{k}}$, by using the information stored in the constraints in $\Phi$, as shown by the following equation: 


$$
\min \left\{f_{p}+\min _{i \in \Phi}\left\{c_{i} \mid \tau_{p} \subseteq U_{i}^{k}\right\}, c_{u_{k}}\right\} \quad \forall k \in\left\{K \backslash K_{f}\right\}, p \in P_{k}
$$

In equation (12), $f_{p}$ denotes the total fixed cost of a path, as determined by the sum of all fixed cost arcs on the path. We calculate an upper bound on the total cost of the path by taking the sum of the smallest upper bound on the total tariff cost of the path and the total fixed cost of the path. Denote the upper bound on the cost of a path $p$ as given in equation (12) by $u\left(c_{p}\right)$. Since the cost of any path $p^{*}$ selected by the client $k \in K$ must be smaller or equal to the cost of all other paths $p \in\left\{P_{k} \backslash p^{*}\right\}$, it must certainly be smaller or equal to $u\left(c_{p}\right)$ for all $p \in\left\{P_{k} \backslash p^{*}\right\}$. Therefore, an upper bound on the revenue for a client is given by:

$$
\min _{p \in P_{k}}\left\{u\left(c_{p}\right)\right\}-c_{l_{k}} \quad \forall k \in K
$$

Hence, the upper bound given in equation (10) can be improved by calculating the total revenue for the operator in a node as follows:

$$
\Pi^{*}+\sum_{k \in K \backslash K_{f}}\left\{\min _{p \in P_{k}}\left\{u\left(c_{p}\right)\right\}-c_{l_{k}}\right\}
$$

Besides improving the upper bound calculation in each node of the branch and bound tree, equation (14) shows that the path for which we expect the highest revenue is still the path with smallest fixed cost.

\subsection{Branching Rules}

In each node of the branch and bound tree, we branch on the paths taken by a client. The selection method of the clients is based on the upper bound on the revenue generated by each client for the operator. The client for which this upper bound is highest, is selected first. Hence, in the root node of the tree we select the client for which we expect the operator to have the highest revenue and branch on all his relevant paths. The smaller the set of relevant paths, the smaller the number of nodes we need to create to fix the path taken by the client. For each of the child nodes of the root node, we select the second highest revenue generating client and branch on all his relevant paths. In this way, for each additional node we create in the tree, we fix the path taken by each additional client. We can continue branching until we have exhausted the set of clients. In the leaves of the tree the path taken by all clients is fixed, whereas in the other nodes some clients still have paths which are not fixed.

\section{Numerical Results}

The branch and bound algorithm (PBB) was implemented in C++, using CPLEX 7.5 to solve the linear programs as described in equation (6) in each node of the tree. The algorithm was tested on data sets which represent telecommunications networks and were provided by France Télécom Research and Development. A description of the data sets used is given in table 1. For each data set, we describe in this table the number of nodes and arcs in the network, the number of tariff arcs and the number of clients.

To illustrate the effectiveness of the reduction methods, table 2 shows some statistics on the number of paths generated for each data set using the shortest paths graph model and its reduction methods. In this table, the column MIN, resp. MAX, indicates the minimum, resp. maximum, number of paths generated over all commodities. The column AVRG gives the average number of paths generated for each commodity for the whole data set. We can see from this table the effectiveness of the shortest paths graph model and its reduction methods by the small number of paths generated on average for the given data sets.

The performance of the PBB algorithm was compared to the mixed integer programming formulation (MIP) of Labbé et al [8] described in section 2, which was implemented in CPLEX 
Table 1: Description of data sets

\begin{tabular}{|l|c|c|c|c|}
\hline Data & \#Nodes & \#Arcs & \#Tariff Arcs & \#Clients \\
\hline D1 & 29 & 92 & 6 & 13 \\
D2 & 29 & 98 & 11 & 13 \\
D3 & 43 & 176 & 10 & 20 \\
D4 & 60 & 212 & 9 & 22 \\
D5 & 60 & 212 & 10 & 8 \\
D6 & 60 & 212 & 17 & 8 \\
D7 & 60 & 212 & 21 & 18 \\
D8 & 49 & 116 & 9 & 23 \\
D9 & 34 & 116 & 15 & 30 \\
\hline
\end{tabular}

Table 2: Paths statistics for each data set.

\begin{tabular}{|c|c|c|c|}
\hline Data & MIN & MAX & AVRG \\
\hline D1 & 2 & 3 & 2.5 \\
\hline D2 & 2 & 5 & 3.1 \\
\hline D3 & 2 & 8 & 5.5 \\
\hline D4 & 2 & 15 & 4.2 \\
\hline D5 & 2 & 20 & 5.0 \\
\hline D6 & 2 & 30 & 12.0 \\
\hline D7 & 2 & 9 & 4.8 \\
\hline D8 & 2 & 10 & 4.0 \\
\hline D9 & 2 & 8 & 3.1 \\
\hline
\end{tabular}

7.5. The computational results for the $\mathrm{PBB}$ algorithm and the MIP were established on an AMD Athlon $800 \mathrm{MHz}$ with $512 \mathrm{Mb}$ RAM, running GNU/Linux with kernel 2.4.8.

Table 3 gives an overview of the results for both algorithms. In this table, the column OPT indicates the value of the optimal solution for the given data set. The column CPU(s), resp. Nodes, indicates the execution time in seconds, resp. the number of nodes in the $\mathrm{B} \& \mathrm{~B}$ tree for each algorithm. For the PBB algorithm, the CPU time includes the time needed for the preprocessing fase of the algorithm. As can be seen in table 3, the PBB algorithm efficiently uses the few relevant paths generated to find the optimal solution for each data set. When we compare its execution to the MIP, we see that the execution time of the PBB algorithm is less or equal to the running time of CPLEX for the MIP for each data set considered. The difference is especially large for the data sets D2, D3, D6, D7 and D8. The most striking difference is for the data set D3, for which the execution time of the MIP is 286358 seconds, whereas the PBB algorithm takes only 137 seconds.

\section{Summary}

In the first part of this paper we studied the complexity of the tariff-setting problem. A variant of the tarification problem, where the set of tariff arcs containing positive flow in the optimal solution are known a priori is shown to be $\mathcal{N} \mathcal{P}$-hard. Another variant, where the tariffs to be determined on all arcs are equal, is shown to be polynomially solvable.

In the second part of the paper we described a method to generate relevant paths for each client in a network. At the core of this method is a graph remodeling, referred to as the shortest paths graph model which, together with powerful reduction methods, generates a sparse graph for 
Table 3: Overview Numerical Results

\begin{tabular}{|c|c|c|c|c|c|}
\hline \multicolumn{2}{|c|}{} & \multicolumn{2}{c|}{ PBB } & \multicolumn{2}{c|}{ MIP } \\
\hline Data & OPT & $C P U(s)$ & Nodes & $C P U(s)$ & Nodes \\
\hline D1 & 6.6225 & 1 & 52 & 5 & 187 \\
\hline D2 & 7.7928 & 1 & 201 & 22 & 1060 \\
\hline D3 & 728.435 & 137 & 14856 & 286358 & 13619345 \\
\hline D4 & 1321 & 158 & 21523 & 540 & 10305 \\
\hline D5 & 995 & 2 & 185 & 3 & 35 \\
\hline D6 & 1426 & 133 & 1195 & 1047 & 71013 \\
\hline D7 & 1565 & 122 & 9689 & 3708 & 76003 \\
\hline D8 & 664406 & 104 & 16709 & 1065 & 35579 \\
\hline D9 & 189180 & 56 & 6129 & 142 & 3141 \\
\hline
\end{tabular}

each client for the tested data sets. Using this sparse graph we are able to generate few relevant paths for each client.

These relevant paths are used in a branch and bound algorithm (PBB) where at each node of the branch and bound tree we solve a simple linear program to obtain lower and upper bounds. The algorithm is compared to the general mixed integer program (MIP) as described by Labbé et al. [8]. It is shown that the PBB algorithm is very effective for the data sets considered when compared to the mixed integer program.

As a possible future extension, the paths generated by the shortest paths graph model and its reduction methods could be used in other models based on a path formulation of the tarification problem. Based on the numerical results given in section 7 , such a path formulation could benefit from the small number of paths generated. Another possible extension is to test the execution of the MIP on the new network created by the sparse shortest paths graph model.

\section{Acknowledgment}

This research was supported by France Télécom Research \& Development.

\section{References}

[1] L. Brotcorne, M. Labbé, P. Marcotte, and G. Savard. A bilevel model and solution algorithm for a freight tariff-setting problem. Transportation Science, 34(3):289 - 302, 2000.

[2] L. Brotcorne, M. Labbé, P. Marcotte, and G. Savard. A bilevel model for toll optimization on a multicommodity transportation network. Transportation Science, 35(4):345 - 358, 2001.

[3] P. Ferrari. Road pricing and network equilibrium. Transportation Research-B, 29B(5):357$372,1995$.

[4] C. Fisk. A conceptual framework for optimal transportation systems planning with integrated supply and demand models. Transportation Science, 20:37-47, 1986.

[5] M.R. Garey and D.S. Johnson. Computers and Intractability: A Guide to the Theory of NP-Completeness. W.H. Freeman, San Francisco, 1979.

[6] R.G. Jeroslow. The polynomial hierarchy and a simple model for competitive analysis. Mathematical Programming, 32:146-164, 1985.

[7] R.M. Karp and J. B. Orlin. Parametric shortest path algorithms with an application to cyclic staffing. Discrete Applied Mathematics, 3:37-45, 1981. 
[8] M. Labbé, P. Marcotte, and G. Savard. A bilevel model of taxation and its application to optimal highway pricing. Management Science, 44:1608-1622, 1998.

[9] L.N. Vicente and P. H. Calamai. Bilevel and multilevel programming: A bibliography review. Journal of Global Optimization, 5:291-306, 1994.

[10] N. E. Young, R. E. Tarjan, and J. B. Orlin. Faster parametric shortest path and minimumbalance algorithms. Networks, 21:205-221, 1991. 